\title{
No Longer an Object: Thermodynamics and New Dimensions of Architectural Design
}

\author{
DORIT AVIV \\ University of Pennsylvania, Princeton University
}

\begin{abstract}
A reconsideration of what constitutes the 'design space' today from the perspective of thermodynamics is necessary in order to expand the realm of architecture into new domains. Building form is dominated in every era by the technological means that produce it. Today, the ubiquitous representations of architecture on computer screens as a context-less object in an empty virtual Cartesian space inherently ignore the constant energetic exchange between a building, the human body and its environment. These exchanges extend not only to the immediate surrounding but all the way to the cosmic scale of the sky and the stars. A pedagogy that engages architecture students with climate and ecology must therefore develop new tools of representation that embody these multi-scalar relationships. In this article, projects produced by students in the design studio are examined as means to both characterize energy flows and intervene on them. As such, they are not just registers of environmental knowledge and sensory data but a first step in redefining the relationship between architecture and the environment.
\end{abstract}

There is a gap in contemporary architectural practice between the design process and the consideration of buildings' energy systems. This is an artificial separation because every design decision has thermodynamic consequences. It stems, in part, from decades-long disciplinary divides fragmenting different aspects of building design to different professionals. Architects have become accustomed to expect of mechanical engineers to provide indoor comfort solutions with solely the plenum space air system in the building. This gap, however, is not only a disciplinary divide; it begins with a pedagogical chasm between building physics research and the work in the design studio.

Debates about the role of science in architectural education date back to the consolidation of professional degrees in architecture. Daniel Ryan (2018) ${ }^{1}$ discusses one such debate in mid-twentieth-century England, in which the RIBA-formed committee the "Architectural Science Group," published in 1941 a report on the Place of Science in Architectural Education, followed by rejection by RIBA's Board of Architectural Education due to "fear that architectural teaching may become a matter of formulae and that the finer points of design will be lost." ${ }^{2}$ The debate reflected the tension between science and art as two poles pulling the discipline in different directions - science deemed to impose "formulae" on the design process, while art was to endow students with creative freedom. Additionally, concerns were raised as to the skillset that architecture students must acquire in order to apply scientific tools in their design methodologies. These questions are ever more prescient today with the explosion of computer simulation software and open-source climate data and environmental data acquisition tools. What role should the application of scientific tools play in contemporary architectural education? How do climate-modeling techniques become generative for the design process rather than imposing constraints? While accredited architectural programs in the United States today are required to teach principles of environmental control systems and sustainability ${ }^{3}$ as part of the core curriculum, the question remains as to how this knowledge influences the work within the design studio.

The design studio is the focal point of a student's creative life in the architectural school, while other courses in the curriculum are, almost always, secondary. Within this hierarchy, any agenda for pedagogical transformation will ramify most fully if it takes place from within the studio itself, an arena that on the other hand, has generally remained resistant to rigorous engagement with ecology and the environmental sciences during most of the previous century. While innovations in the field of structural engineering were arguably the driving force of form-making for modernist architects in the early twentieth century, ${ }^{4}$ despite the environmental turn ${ }^{5}$ and rising awareness of the significance of energy utilization in buildings and its ecological consequences, mechanical engineering has not risen to a comparable status as an architectural form-maker.

In Climates: Architecture and the Planetary Imaginary (2015), the editors ask "what are the material and conceptual infrastructures that render climate legible, knowable, and actionable, and what are their spatial implications?" ${ }^{\prime}$ As this question implies, it is hard to pinpoint a clear formal vocabulary that could make the climatic performance of buildings legible, knowable and actionable. This is first, because of the invisibility of heat transfer and second, because of the historical treatment of mechanical systems. Per the twentieth century paradigm, mechanical systems of chillers, ducts and vents are treated by architects as black-boxes, untouched and unseen. As argued in Robert Venturi's Complexity and Contradiction (1977), 7 their spatial expression in modern architecture has been contained in the building's "poché space," i.e. within wall cavities and dropped ceilings. We have become accustomed to think of heat transfer as lacking a 


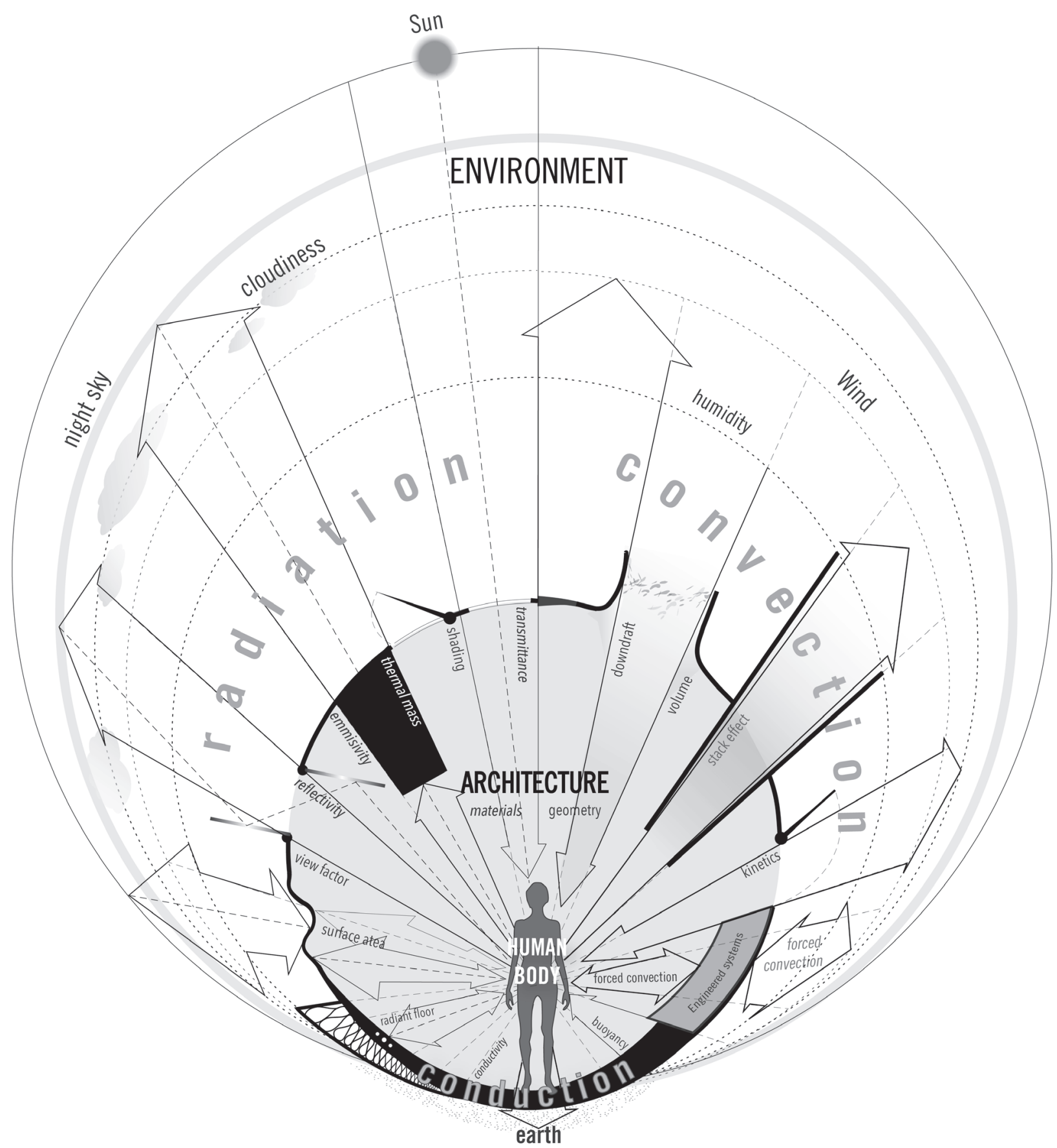

Figure 1. Diagram of thermodynamic influences: the exchange of heat between humans and the environment is dominated by mainly radiation and convection, while conduction is only responsible for a small percentage of energy transfer from the body. Architecture acts a mediator in this exchange as design elements instigate or disable the transfer of heat from the human body to its surroundings (cooling), or vice versa (heating), as indicated by the diagram's arrows. Their material properties and geometric configuration define the nature of the thermodynamic process they generate. In every architectural design it is possible to utilize one or many modes of cooling and heating from this array of possibilities. Drawing by author. 
defined form. What I propose here, as a new model, is neither an exposition of the mechanical space hidden in the poché, such as in Piano and Roger's Centre Pompidou, nor a continuation of the conventional architectural engagement with heat transfer limited to the R-value of the wall construction, but rather a reconsideration of the surfaces and interior volumes of the architectural object as active parts of the dynamics of heat transfer.

Through the dissemination of digital modeling tools during the past three decades, the ubiquitous representation of the architectural object is as surface geometry, autonomous and isolated in an abstract digital space. However, in the physical environment, an architectural surface is constantly emanating and absorbing heat through radiation exchange with the sun, the sky, the surface of human bodies, and other surrounding inanimate matter. Not only surfaces, but the entirety of the building's interior rooms and cavities are sites for thermal interactions, where hot and cold flows of air react to the geometric and material properties of the volumes through which they move (figure 1). While conduction occurs only between objects which touch each other, convection transports heat through air motion affected by atmospheric conditions. Thermal radiation - being a spectrum of light - exchanges heat at various wavelengths at all distances - as close as a hand's reach and as far as the sun. Heat transfer physics thus connects architectural surfaces and volumes to a much larger ecosystem. At the same time, it centers the design process on the human being and is therefore inherently humanistic: it places an emphasis on embodied thermal sensation that is inevitably experienced from an individual perspective. This sensation is both psychological and physical, dependent on every characteristic of our traits and behavior, from total surface area, to level of clothing and activity (figure 2).

In "An Ecology of Bodies" (2015) Ross Exo Adams poses that a contemporary view of architecture, from the perspective of its interaction with climate, shifts our notion of "scale" and "object" into "hyperobjects," which traverse our traditional scalar and temporal categories. Especially the urban, he argues, surpasses all traditional scales and spatial confines $^{8}$. Adams writes:

From "metropolitan" to "megalopolitan" to "regional" to "planetary" in scale, urbanization now reveals itself traversing deserts, arctic regions, and can even be found in the atmosphere. Indeed, it is possible that we can no longer comprehend the urban as a spatiality isolated to any one particular scale at all but must instead illuminate it in its full, trans-scalar ontology. ${ }^{9}$

These scalar relationships are explored in the following student work by James Seung Hwan Kim (figure 3) where the typology of the urban tower is revisited in relation to not only the urban context but to regional and global wind patterns.

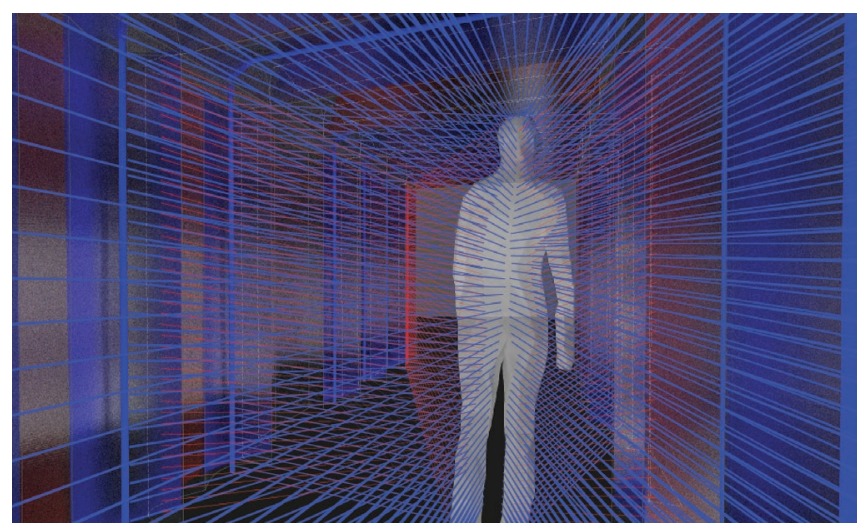

Figure 2A. Illustration of radiant fluxes from the body to its surrounding hot and cold surfaces.

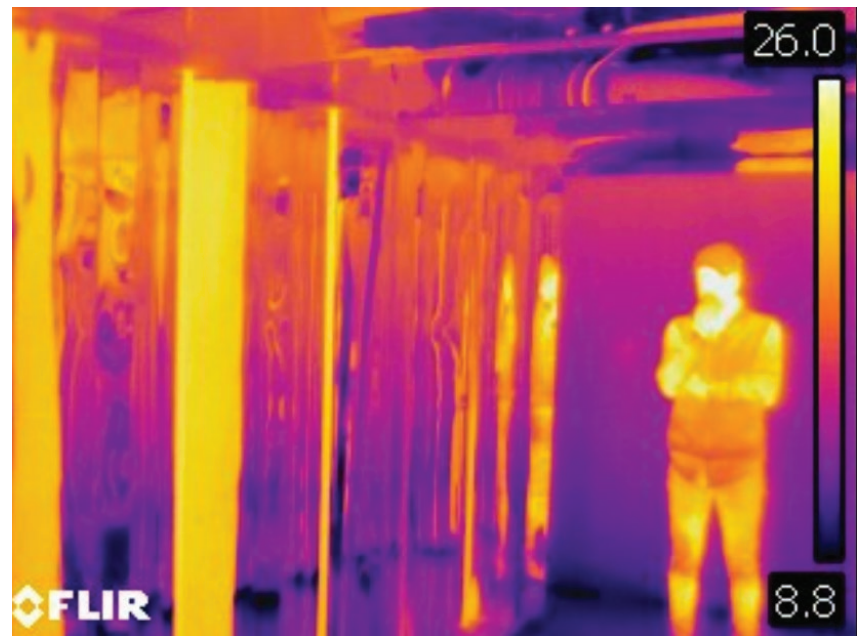

Figure $2 \mathrm{~B}$. Infrared image of the hysical prototype built to test the radiant exchange simulated in image $2 \mathrm{~A} .{ }^{10}$ Images by Dorit Aviv, Melanie Daguin, Forrest Meggers and Eric Teitelbaum.

This chain of links between different scales is in fact necessary when pertaining to wind, which is both continuous in space and yet abruptly changing and stochastic in time because of its dependence on multiple constantly shifting factors. Through its interaction with built form, the magnitudes of the forces driving airflow are locally enhanced and multiplied or conversely subdued dramatically. Due to conservation of mass and momentum, when this happens, the inverse effect will occur elsewhere. Thus in an urban environment, airflows and energy flows inevitably link buildings into a tightly-knit mutually dependent environmental fabric. To search for a skyscraper form that produces a certain microclimate through its interaction with airflow, it was therefore necessary to expand the design to the scale of the block, the city, and the globe.

The dependence of thermodynamics on the spatial characteristics of buildings invites designers to intervene on the energy performance of buildings using the most fundamental tools 
at an architect's disposal: geometry and materials. In fact, the advancements in digital representation of architectural geometry can be used precisely for this purpose. As surveyed in Pottman et al (2007), ${ }^{11}$ contemporary computational tools discretize and parameterize space. They not only enable the rationalization and fabrication of forms that were previously not considered constructible, but also re-configured the design process itself and the conception of curve, surface,
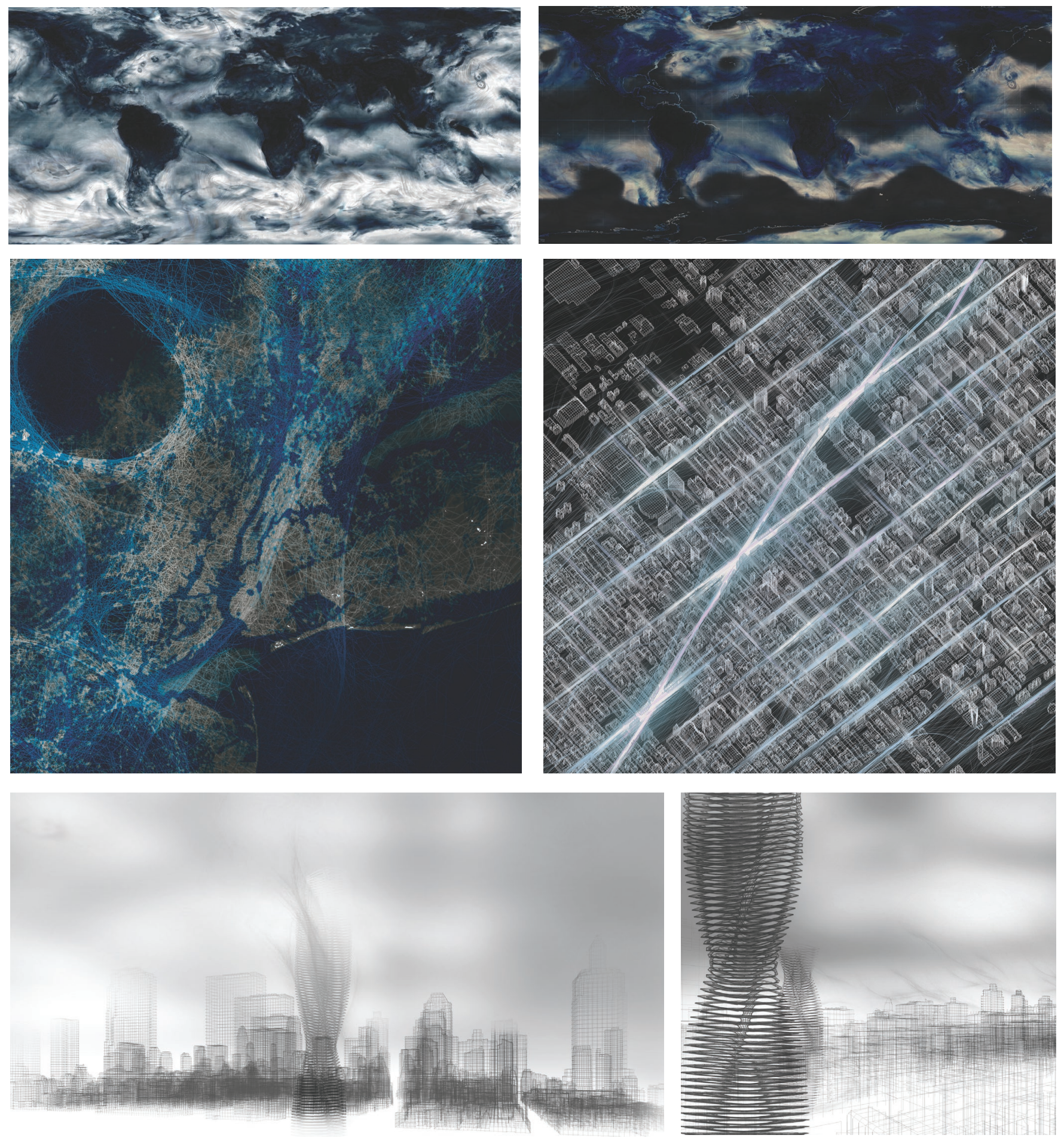

Figure 3. Manhattan Wind Skyscraper project by James Seung Hwan Kim, showing scalar relationship between the global, regional, urban and architectural. Fall 2015 Master's Studio at The Cooper Union School of Architecture Studio. Instructors: Pablo Lorenzo-Eiroa, Dorit Aviv and Will Shapiro. Images courtesy of the the irwin S. Chanin school of Architecture Archive. 
and volume. By extension, we now use these advanced analytical tools, together with computational simulation tools to better understand heat transfer.

From packaged programs such as Autodesk Ecotect to opensource tools such as Ladybug and Honeybee ${ }^{12}$ plugins to Grasshopper Algorithmic Modeler, simulation software has made huge contribution to the ability of architects to obtain climate analysis, especially radiation analysis of buildings. However, to most users, they remain black-boxes producing numerical results and color gradients. Unless a designer using solar heat gain simulation is familiar with solar geometry and the structure of the software code, then the actual mechanism producing the results -utilizing vector intersection with discretized mesh test-points based on weather-file numerical data- remains opaque. My pedagogical position is that student work should "break the black-box," by producing both physical and digital climatic simulations from first principles. The following drawings by the Environmental Building Design students at the University of Pennsulvania (figure 4), offer a not only a visualization of energy flows in urban space, but also open the gate to purposeful intervention on them: if one is able to represent the geometry of invisible physical phenomenon, then one can understand what might influence it and divert its track. While this specific project was not produced for solar radiation, its technique is similar to urban radiation simulations.

In the indoor environment, a person walking inside of a room in fact thermally performs like a sun: they emit heat to their surrounding surfaces, creating a field of thermal events around them. But unlike solar radiation, radiation from the body is diffuse and is geometrically characterized accordingly (figure 2). The human body is a complex thermal engine, with multiple sophisticated mechanisms for exchanging heat with its environment. Intuitively, we only perceive this exchange through our bodily thermal sensations in space. It is clear to us that as we move through any environment we might feel cold or hot at different moments depending on our own metabolic rate and our exposure to external forces. Yet it is still rare for designers to use thermal sensations as part of the toolkit for space-making. This again revolves around the difficulty of representation of thermal sensation in architectural drawing. The following student project by Graham Nelson (figure 5a) tackles this problem. In his drawings, he used mean radiant temperature simulations in 3D space and turned them into thermal clouds through which people move.

In parallel, the explosion of real-time sensing tools today offers an opportunity to learn directly from the physical artifact. The results of a design hypothesis can be measured, assessed, and revised, effectively collapsing the laboratory space into the design studio. Figures $2 \mathrm{~b}$ and $5 \mathrm{~b}$ show thermal imaging of physical prototypes used to determine design parameters. One of the main challenges of integrating scientific methods into architecture pedagogy is that student designs remain hypotheses only, never tested for their performance in the physical world. By contrast, environmental science is aimed towards performance in real world application and innovations in the field must provide proof of their functionality. Physical prototypes with live sensor feedback offer the opportunity to test design hypotheses. This is an important shift from a design pedagogy that focuses on digital representations only, where fabrications are only a means to realize the imagined virtual construction, into a design space that includes the behavior of the built prototype not as an artifact alone but as a field of study in itself. In turn, new forms of representation emerge from the processing of thermal data (thermography is but one example), and enrich the architectural vocabulary.

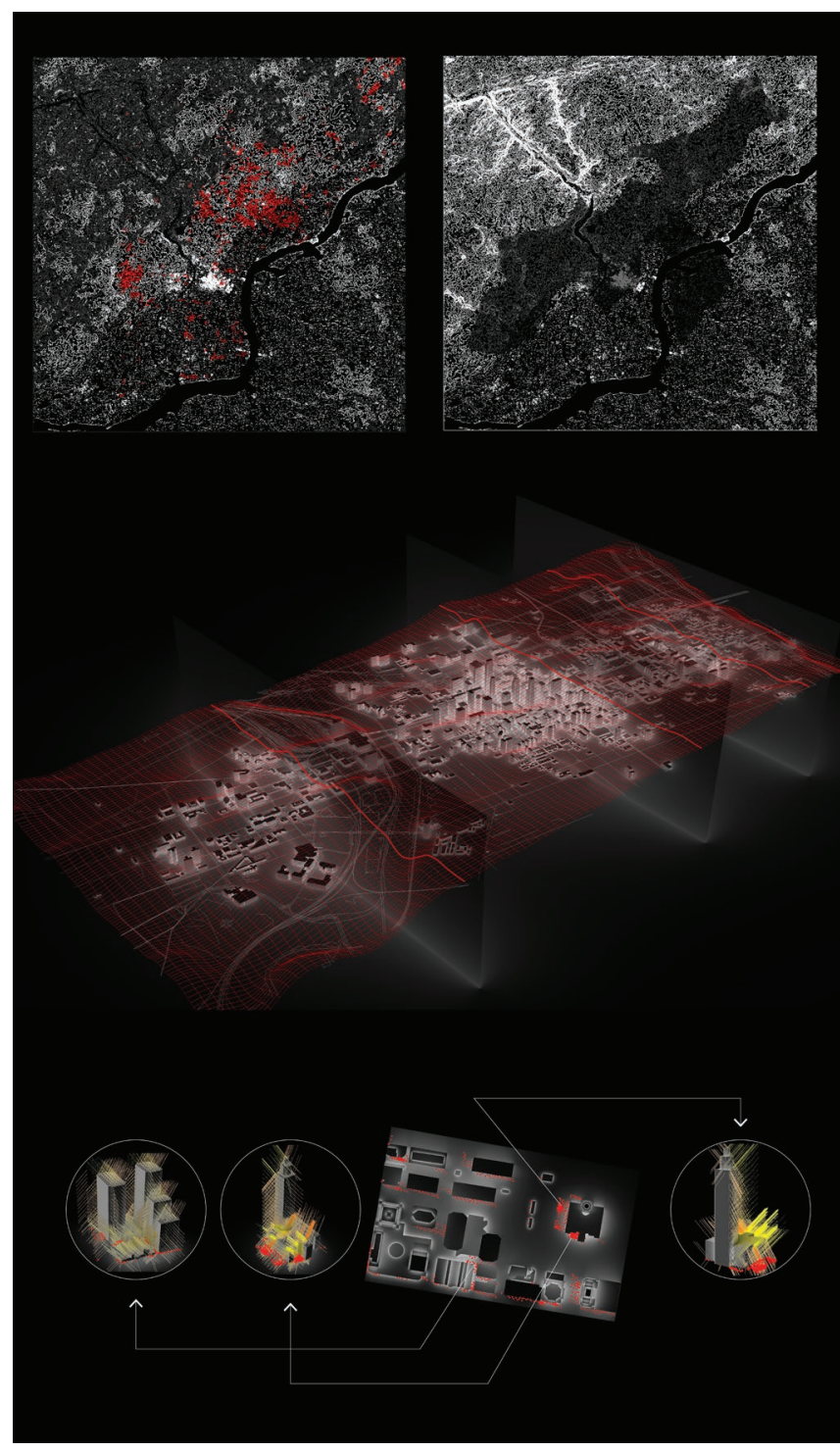

Figure 4. Analysis of Philadelphia downtown climate based on radiation simulation and ray-tracing ${ }^{12}$ as well as GIS-based surface temperature maps. ${ }^{13}$ Drawings by Chunyi Wang, Liang Zhang and Yunwen Zhu. Studio taught in 2019 at the University of Pennsylvania School of Design by Dorit Aviv and Kit Elsworth. 


\section{ACKNOWLEDGEMENTS}

I'd like to acknowledge the influential pedagogical work by Pablo Lorenzo-Eiroa who coordinated the 2015 Studio at The Cooper Union

\section{ENDNOTES}

1 D. Ryan, "Architects in White Coats," in Sydney School: Formative Moments in Architecture, Design and Planning at the University of Sydney, eds., Andrew Leach and Lee Stickells (Melbourne: Uro, 2018), 71-90.

2 Ryan, 71-90.

3 National Council of Architectural Registration Boards, Education Guidelines 2018, accessed January 2019. https://www.ncarb.org/become-architect/ study-architecture.

4 Henry J. Cowan, An Historical Outline of Architectural Science (London: Applied Science Publishers, 1977).

5 Adam Rome, “'Give Earth a Chance:' The Environmental Movement and the Sixties," The Journal of American History 90, no. 2 (2003): 525-554.

6 James Graham, et al., "Climates: Architecture and the Planetary Imaginary," Climatic Change 93 (2009): 335-354.

7 Robert Venturi, Complexity and Contradiction in Architecture, Volume 1 (New York: The Museum of Modern Art, 1977).
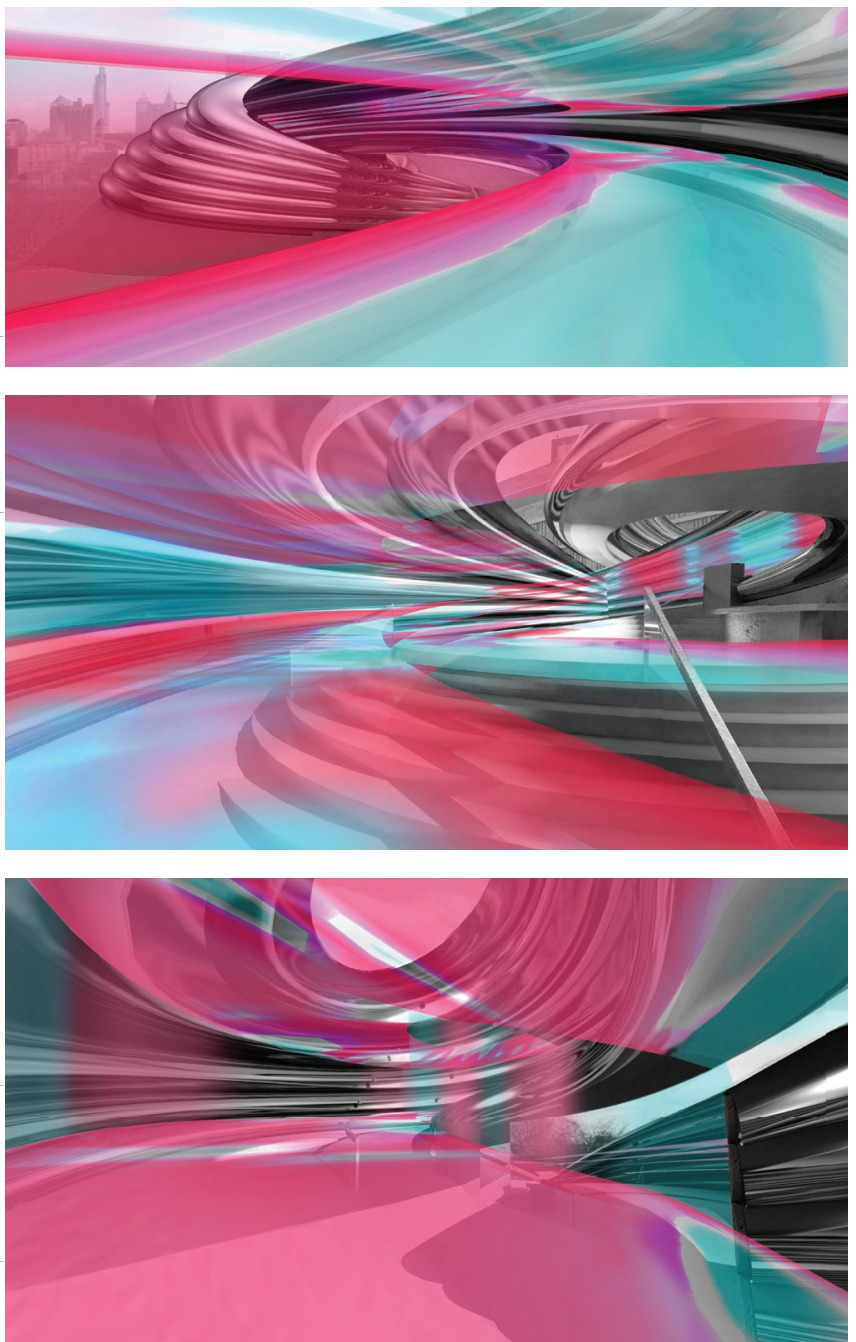

8 Ross Exo Adams, "An Ecology of Bodies," Climates: Architecture and the Planetary Imaginary (2015): 181-190.

9 Adams, 181.

10 Images from the installation at the 2017 Seoul Biennale for Architecture and Urbanism by Forrest Meggers and Dorit Aviv "Energy is Everywhere and Nowhere," Imminent Commons: Urban Questions for the Near Future: Seoul Biennale of Architecture and Urbanism 2017, eds., Alejandro Zaera-Polo and Jeffrey S. Anderson (Barcelona: Actar, 2017).

11 Helmut Pottmann, Andreas Asperl, Michael Hofer, and Axel Kilian, Architectural Geometry Volume 724 (Exton: Bentley Institute Press, 2007).

12 Mostapha Sadeghipour Roudsari, Michelle Pak, and Adrian Smith. "Ladybug: A Parametric Environmental Plugin for Grasshopper to Help Designers Create an Environmentally-Conscious Design," in Building Simulation 2013: 13th International Conference of the International Building Performance Simulation Association (International Building Performance Simulation Association, 2013).

13 Ken Steif, "Urban Heat Islands \& Street Trees in Philadelphia," Urban Spatial official website, accessed January 2019. http://urbanspatialanalysis.com/ urban-heat-islands-street-trees-in-philadelphia/.
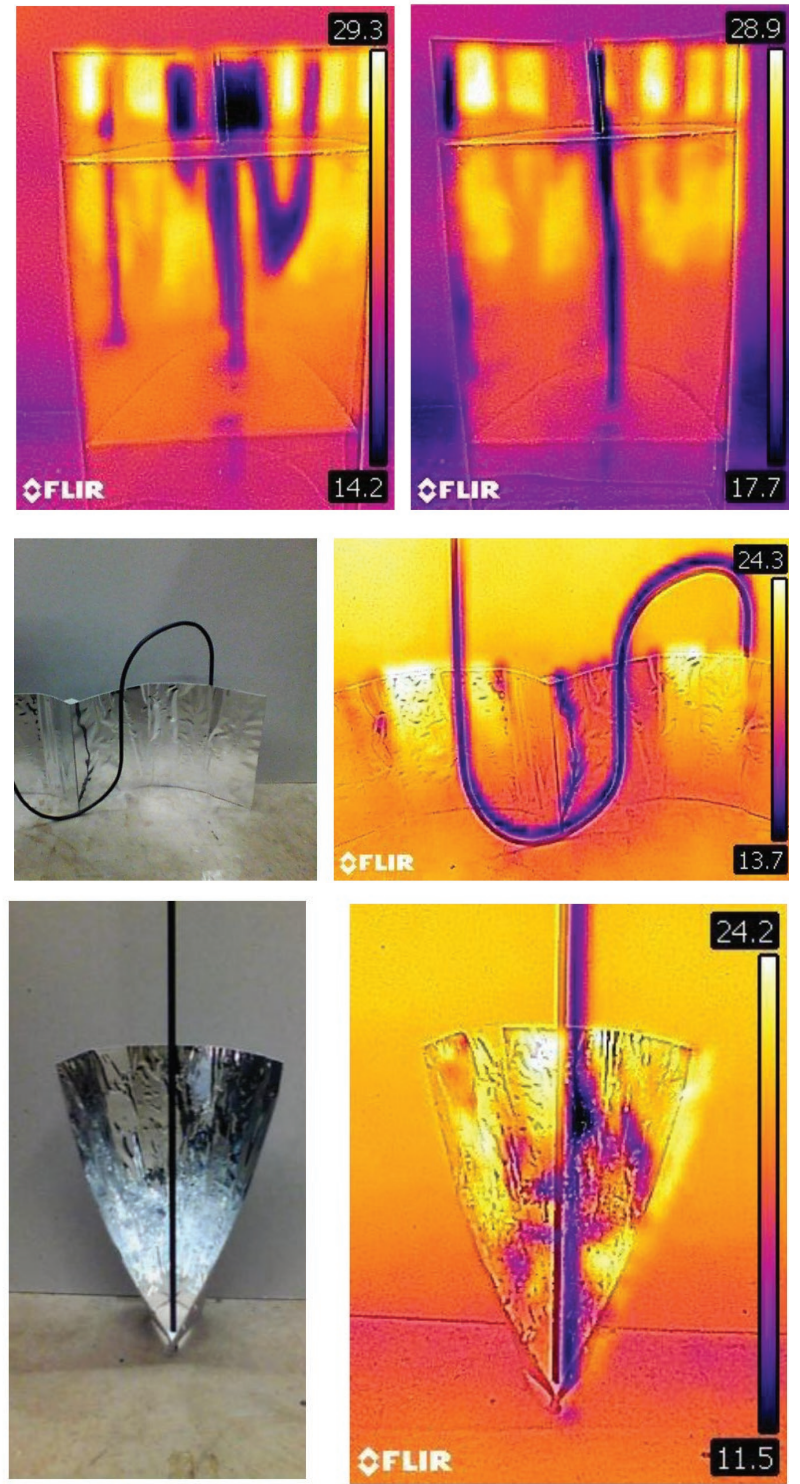

Figure 5. drawings and photographs by Graham Nelson, produced in Fall 2018 MEBD studio at the University of Pennsylvania School of Design. Instructor: Dorit Aviv. Left, 3D-mean radiant temperature cloud renderings; right, small-scale prototype tests with infrared camera. 